\title{
Utilidad de la detección de células citomegálicas circulantes en pacientes inmunosuprimidos VIH negativos en el control de la infección por citomegalovirus
}

\author{
María Eugenia Castaño ${ }^{1}$, Mauricio Sarmiento ${ }^{1}$, Lázaro Vélez ${ }^{2}$, Juan Carlos González ${ }^{1}$, \\ Francisco Cuéllar ${ }^{3}$, María Teresa Rugeles ${ }^{1}$,Victoria Inés Bedoya ${ }^{1}$ \\ ${ }^{1}$ Grupo de Inmunovirología, Corporación Biogénesis, Universidad de Antioquia, Medellín, Colombia. \\ ${ }^{2}$ Grupo Investigador de Problemas en Enfermedades Infecciosas (GRIPE), Facultad de Medicina, Universidad \\ de Antioquia, Medellín, Colombia. \\ ${ }^{3}$ Unidad de Trasplantes de Médula Ósea, Facultad de Medicina, Universidad de Antioquia, Medellín, Colombia.
}

Objetivo. Buscar la correlación entre la presencia de células citomegálicas circulantes con la antigenemia pp65 para citomegalovirus y el desarrollo de complicaciones clínicas inherentes a infección y enfermedad por citomegalovirus.

Materiales y métodos. Entre diciembre de 2002 y julio de 2003 se procesaron 110 muestras de sangre periférica, obtenidas de 46 pacientes inmunosuprimidos. La antigenemia pp65 y la presencia de células citomegálicas circulantes se determinaron mediante inmunofluorescencia indirecta utilizando un estuche comercial para la detección del antígeno pp65 de citomegalovirus. En leucocitos de sangre periférica, la antigenemia fue positiva cuando se encontró una o más células con tinción fluorescente, multilobulada y homogénea en el núcleo celular. Se determinó la presencia de células citomegálicas circulantes cuando se observaron células de gran tamaño (35 a $50 \mathrm{~mm}$ ) con un patrón de tinción fluorescente extendido en todo el citoplasma celular en células mononucleares de sangre periférica.

Resultados. Se encontraron ocho pruebas con antigenemia positiva, procedentes de siete pacientes (15\%). De éstos, cuatro $(57 \%)$ también presentaron células citomegálicas circulantes, tres habían recibido un trasplante renal y uno un trasplante hepático. El número de células positivas en la antigenemia fue mayor en los pacientes con trasplante renal que en el resto de pacientes inmunosuprimidos (457 vs.1,96; $p<0,005)$. No se encontró asociación entre la presencia de células citomegálicas circulantes, la morbilidad, la mortalidad y el desarrollo de enfermedad injerto contra huésped (GVHD) $(p<0,001)$.

Discusión. No encontramos correlación entre la detección de células citomegálicas circulantes, la antigenemia y la mortalidad en estos pacientes. Sin embargo, es necesario realizar estudios prospectivos con un mayor número de individuos para determinar mejor dicha correlación y definir su utilidad clínica en pacientes inmunosuprimidos.

Palabras clave: citomegalovirus, antigenemia, células citomegálicas circulantes, inmunosupresión.

Presence of circulating cytomegalic cells in HIV negative immunosuppressed individuals following cytomegalovirus infection

Objective. Immunofluorenscence methods to detect pp65 antigenemia were implemented for identifying the circulating virus-infected cells in individuals known to have cytomegalovirus infection and disease symptoms.

Material and methods. Between December-2002 and July-2003, 110 peripheral blood samples were obtained from 46 immunosuppressed patients. pp65 antigenemia and the presence of circulating cells were determined by indirect immunofluorescence using a commercial kit to detect CMV pp65 antigen in peripheral blood leukocytes. Antigenemia was positive when one or more cells was observed with multilobulated, homogenous fluorescent stain in the nucleus. The presence of infected circulating cells (peripheral blood mononuclear cells) was determined when an extended pattern of fluorescent stain was observed throughout the cytoplasm in cells of 35 to $50 \mu \mathrm{m}$. 
Results. Eight antigenemias from 7 patients (15\%) were positive. Of these, 4 (57\%) were also positive for circulating infected cells and consisted of 3 kidney transplant recipients and 1 liver transplant recipient. The number of positive cells in antigenemia was greater in kidney-transplant recipients than in the rest of immunosupressed patients (457 vs.1.96, $\mathrm{p}<0.005)$. No association was seen between the presence of infected circulating cells, morbidity, mortality or the development of GVHD $(p<0.001)$.

Conclusion. No correlation was observed between the presence of infected cells, antigenemia and mortality. To substantiate the lack of correlation amongst these factors, prospective studies with larger sample sizes are necessary. These studies will aid in better defining the clinical application in immunosupressed patients.

Keywords: cytomegalovirus, antigenemia, cytomegalic circulating cells, immunosuppression.

La infección por citomegalovirus (CMV) es altamente prevalente en los países en desarrollo; se calcula que $70 \%$ a $100 \%$ de sus adultos tienen evidencia serológica de haberse infectado con anterioridad pero, generalmente, se asocia con enfermedad sólo en los individuos inmunodeficientes. La infección primaria por CMV ocurre por contacto directo con líquidos corporales de un individuo infectado, como también por las vías transplacentaria, perinatal y sexual. En los individuos competentes inmunológicamente, los virus permanecen en estado latente o persisten como una infección crónica con bajo nivel de replicación $(1,2)$. Sin embargo, en los pacientes inmunosuprimidos, como en los trasplantados de médula ósea y de órganos sólidos y en pacientes con el síndrome de inmunodeficiencia adquirida, el CMV es uno de los patógenos oportunistas que ocasiona mayores tasas de morbilidad y mortalidad (1-3). Quienes enferman por CMV, generalmente, sufren de neumonitis grave, pero también pueden presentar gastroenteritis, hepatitis y retinitis (1). A pesar de que la terapia para tratar los pacientes con enfermedad por CMV es a menudo efectiva (ganciclovir e inmunoglobulina anti-CMV), la morbimortalidad continúa siendo alta.

Como no es posible hacer un diagnóstico clínico preciso de infección y enfermedad por CMV, se utilizan pruebas virológicas sensibles y rápidas para el diagnóstico oportuno de la infección activa.

\section{Correspondencia}

Victoria Inés Bedoya, Carrera 53 No. 61-30, piso 5, torre 2, laboratorio 532, Laboratorio de Inmunovirología, Facultad de Medicina, Universidad de Antioquia, Medellín. Colombia. Teléfono: 210 6485; fax: 5106047

victoriabedoya@eudoramail.com

Recibido: 27/07/04; aceptado: 09/12/04
Entre éstas se encuentran la viremia, la antigenemia pp65, la PCR y la detección de células endoteliales citomegálicas (4-7). Para evaluar la presencia de replicación activa del virus en receptores de trasplantes y en pacientes con neoplasias y otras enfermedades hematológicas inmunosupresoras, se han definido puntos de corte para cada una de las diferentes pruebas, por encima de los cuales es más probable que aparezca la enfermedad: 10 células infectadas por cada 150.000 leucocitos de sangre periférica inoculados en cultivo celular para la viremia; 100 leucocitos infectados por cada 150.000 leucocitos para la antigenemia pp65, y 1.000 copias de ADN viral en leucocitos para la concentración de ADN viral en sangre, determinada por PCR, mientras que la presencia de una sola célula endotelial citomegálica es suficiente para sugerir enfermedad activa diseminada por CMV con compromiso de órganos (6).

Los pacientes con infección activa por CMV pueden presentar daños en el funcionamiento de los órganos afectados, aún sin síntomas clínicos de la enfermedad. Aunque los mecanismos fisiopatológicos inducidos por el virus no son claros, se cree que las células endoteliales están implicadas pues, por estudios histopatológicos, se ha determinado que estas células son blanco importante del CMV, junto con las células epiteliales y las células de músculo liso (8-10); además, se han encontrado en sangre periférica durante el pico de antigenemia pp65 -o un poco después- en pacientes con infección activa por CMV $(1,4,9,11-14)$.

En células endoteliales citomegálicas se han identificado proteínas virales de origen temprano, inmediatamente temprano y tardías, lo que indica 
infección activa $(4,12,15)$. La presencia de estas células en sangre periférica puede también estar relacionada con la gravedad de la enfermedad por CMV y el compromiso de órganos $(4,12,16,17)$. Aunque no se ha podido confirmar la relación entre los síntomas clínicos y la presencia de células endoteliales citomegálicas en sangre $(4,17)$, se ha demostrado que su presencia se asocia con ausencia o insuficiencia del tratamiento anti-CMV, o con la aparición de cepas resistentes al tratamiento antiviral. También se asocia con la duración de la enfermedad en el órgano afectado $(4,5)$.

Por todo lo anterior, se ha propuesto que la detección de células citomegálicas circulantes sirve como un nuevo parámetro disponible para determinar la actividad de la enfermedad y la gravedad de la misma, para diagnosticar compromiso de órganos por CMV y para el estudio temprano de la patogénesis de infecciones diseminadas por CMV $(4,12)$. El objetivo de este estudio fue correlacionar la presencia de células citomegálicas circulantes en sangre periférica con el resultado de la antigenemia pp65 y la presencia de enfermedad o complicaciones asociadas a $\mathrm{CMV}$ en pacientes inmunosuprimidos.

\section{Materiales y métodos}

\section{Pacientes}

Entre diciembre de 2002 y julio de 2003 se procesaron en el Laboratorio de Virología de la Facultad de Medicina de la Universidad de Antioquia, 110 muestras de sangre periférica para la detección de células citomegálicas circulantes y antigenemia pp65 para CMV. Estas muestras se obtuvieron de 46 pacientes inmunosuprimidos que, de acuerdo con el criterio del médico tratante, requerían evaluación de la infección por CMV. Estos pacientes aceptaron voluntariamente participar en el estudio según consta en el consentimiento informado; el estudio fue aprobado por el Comité de Ética de la Facultad de Medicina de la Universidad de Antioquia.

Según el resultado de la antigenemia se evaluaba la necesidad de realizar pruebas de seguimiento. Los datos demográficos y de evolución de los pacientes se obtuvieron de las historias clínicas.
El tratamiento farmacológico para los pacientes con infección activa por CMV fue determinado por el equipo médico a cargo, de acuerdo con los protocolos establecidos para el manejo de cada enfermedad.

\section{Prueba de antigenemia para CMV}

Las muestras se procesaron con un estuche comercial (CMV BriteÔ, IQ Corporation, Netherlands) para la detección del antígeno pp65 de CMV en leucocitos de sangre periférica según las instrucciones de los fabricantes. Los polimorfonucleares neutrófilos (PMN) se obtuvieron a partir de muestras de $5 \mathrm{ml}$ de sangre venosa tratadas con EDTA, luego de separarlos por sedimentación con dextrano. Para asegurar la pureza de las células blancas, se realizó lisis de eritrocitos con cloruro de amonio al $0,8 \%$; los leucocitos se lavaron dos veces con solución salina amortiguada con fosfatos (PBS); luego, las células se cuantificaron en hemocitómetro y se ajustó su concentración a $1,5 \times 10^{6}$ células por mililitro; de la dilución anterior se tomaron $100 \mathrm{ml}(150.000$ células) y se procesaron en citocentrifuga (Cytospin 3, Shandon Corporation, USA) a 900 revoluciones por minuto durante 3 minutos en placas recubiertas con teflón (Shandon Corporation, USA); las muestras se dejaron secar a temperatura ambiente, se fijaron con paraformaldehído al $20 \%$ durante 10 minutos y se lavaron con PBS durante 5 minutos; luego, fueron permeabilizadas con Nonidet P-40 y lavadas con PBS durante 5 minutos; posteriormente, se añadieron $35 \mu \mathrm{l}$ de anticuerpo monoclonal de ratón contra la proteína pp65 a los pozos de teflón que contenían las muestras; todas las muestras se procesaron por duplicado. Las placas se incubaron durante 30 minutos a $37^{\circ} \mathrm{C}$ y se lavaron dos veces con PBS durante 2 minutos en cada lavado, para retirar los restos de anticuerpo no utilizado. Después de esto, se añadieron $35 \mathrm{ml}$ del anticuerpo antiglobulina de carnero conjugado con FITC y azul de Evans a cada pozo. Las placas se incubaron durante 30 minutos a $37^{\circ} \mathrm{C}$ y se lavaron dos veces con PBS por 2 minutos cada lavado. A cada placa se le agregó glicerol como medio de montaje y se observaron en un microscopio de epifluorescencia (Nikon ${ }^{\circledR}$, Japón) a 250x. 
El resultado se consideró positivo cuando se encontraron una o más células con tinción amarillo-verdosa (fluorescente), multilobuladas y homogéneas en el núcleo celular (figura 1), y negativo cuando las células no presentaron esta tinción. Como control positivo se utilizaron las placas suministradas por el fabricante del estuche, que contienen células pp65 positivas mezcladas con leucocitos antígeno-negativos.

\section{Detección de células citomegálicas circulantes}

La detección de las células citomegálicas circulantes se realizó según la técnica descrita previamente (5). Brevemente, se obtuvo la fracción de células mononucleares a partir de $10 \mathrm{ml}$ de sangre total, mediante gradiente de FycollHypaque (densidad 1.077, Sigma-Aldrich Corporation, St. Louis, MO, USA). Una vez obtenidas las células, se ajustó su concentración a $1,5 \times 10^{6}$ células por mililitro; de la dilución anterior se tomaron $100 \mathrm{ml}$ (150.000 células) y se procesaron en citocentrífuga. Luego, se continúo el proceso de lavado, tinción con anticuerpos, incubación y montaje, de la misma manera como se describió para la prueba de antigenemia.

La prueba se consideró positiva cuando se observó, en células de gran tamaño (aproximadamente, 35 a $50 \mu \mathrm{m}$ ), un patrón de tinción fluorescente de color verde extendido en todo el citoplasma celular (figura 1).

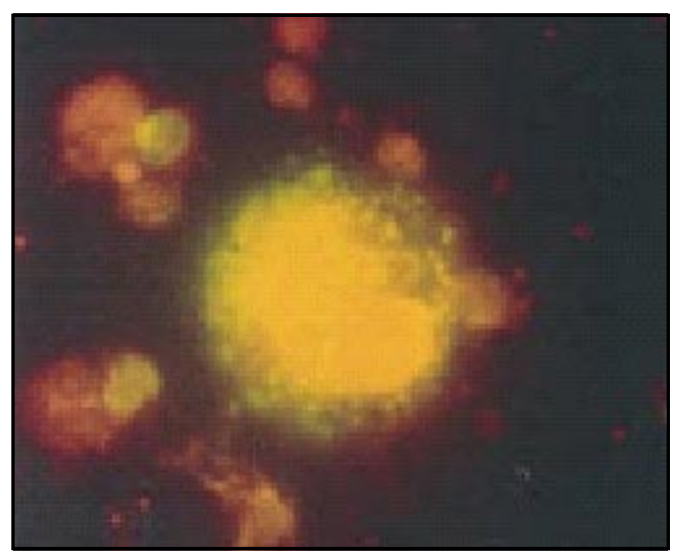

A. Célula citomegálica circulante

\section{Análisis estadístico}

Los datos se almacenaron en una base de datos elaborada con el programa Microsoft Excel ${ }^{\circledR}$ y se analizaron con el programa Epilnfo $2000^{\circledR}$. Los resultados se expresaron como medias, frecuencias y porcentajes. Para los análisis de cruces de las variables categóricas se utilizaron la prueba exacta de Fisher y la prueba de ji al cuadrado. Se aceptaron valores de $p<0,05$ como estadísticamente significantes.

\section{Resultados}

Durante el período de estudio se determinó la presencia del antígeno pp65 de CMV en leucocitos de sangre periférica y la presencia de células citomegálicas circulantes en 46 pacientes inmunosuprimidos atendidos en el Hospital Universitario San Vicente de Paúl y de la Clínica Las Américas de Medellín. La edad promedio fue de 28 años (rango, 2 a 69) y la relación de sexo (masculino/femenino) fue de $25 / 21$. Los trasplantes, tanto de órgano sólido (25 pacientes) como de médula ósea (16 pacientes), fueron las condiciones inmunosupresoras más comunes. Cinco pacientes sufrían leucemia linfoide aguda y uno, anemia aplástica.

Se realizaron 110 ensayos de laboratorio para establecer la presencia de antígeno pp65 y células citomegálicas circulantes (promedio de muestras por paciente: 2,37; rango: 1 a 14). Se encontraron

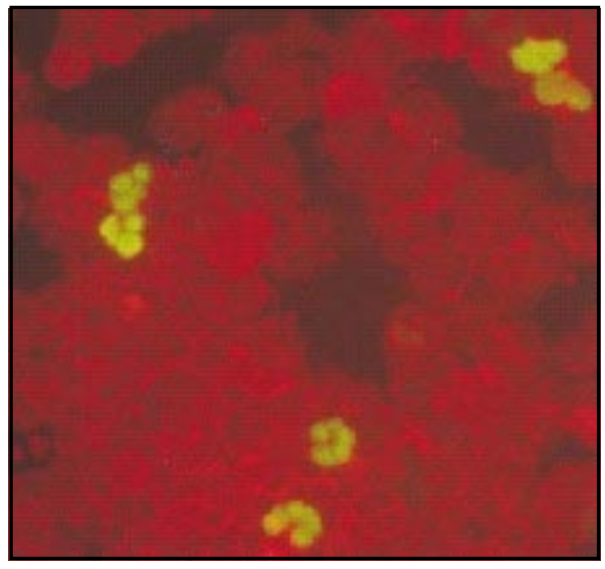

B. Antigenemia positiva

Figura 1. Patrón de fluorescencia de células citomegálicas circulantes y de células pp65 positivas. A. Células de 35 a 50 micrómetros con tinción fluorescente de color verde en todo el citoplasma celular, 1.000X. B. neutrófilos con tinción amarilloverdosa (fluorescente), polilobulada y homogénea en el núcleo celular, 400X. 
Cuadro 1. Relación entre la presencia de células citomegálicas circulantes y antigenemia para pp65 de CMV.

\begin{tabular}{lcc}
\hline & Antigenemia $\mathbf{C M V}^{1}$ & Células citomegálicas circulantes $^{2}$ \\
\cline { 2 - 3 } Paciente 1: trasplante renal & 317 & 2 \\
Paciente 2: trasplante hepático & 40 & 2 \\
Paciente 3: trasplante renal & 425 & 1 \\
Paciente 4: trasplante renal & 872 & 2 \\
Paciente 5: trasplante de médula ósea & 2 & 0 \\
Paciente 6: trasplante renal & 1 & 0 \\
Paciente 7: trasplante renal & 600 & 0
\end{tabular}

${ }^{1}$ PMN positivos en 150.000 leucocitos analizados

${ }^{2}$ Células citomegálicas circulantes en 150.000 leucocitos analizados

${ }^{3}$ Trasplante de medula ósea

* Este paciente presentó 2 antigenemias positivas durante su evaluación con el mismo valor. Las pruebas realizadas como seguimiento fueron negativas una vez instaurado el tratamiento médico.

ocho pruebas de antigenemia positivas que procedían de siete pacientes (15,2\%). De éstos, cuatro $(57 \%)$ también presentaron células citomegálicas circulantes. No se presentó ningún caso de hallazgo de células citomegálicas circulantes en ausencia de antigenemia positiva (cuadro 1).

Se observó que los pacientes con trasplante renal presentaron mayores niveles de células positivas por la antigenemia que los pacientes con otro tipo de trasplante o inmunosupresión debida a otra enfermedad (promedio de células positivas: 457 vs. 1,$96 ; p<0,005$ ). Aunque todos los pacientes que presentaron células citomegálicas circulantes también presentaron antigenemia positiva, no todos los pacientes con antigenemia positiva presentaron células citomegálicas circulantes $(p>0,05)$. No se observó asociación estadística entre la presencia de células endoteliales y el número de células positivas en la antigenemia.

Ninguno de los pacientes presentó enfermedad por CMV. Si bien algunos pacientes presentaron procesos febriles y compromiso pulmonar, gastrointestinal o neurológico, siempre se demostró otra causa, como infecciones bacterianas y fúngicas, o complicaciones propias de los tratamientos realizados como la enfermedad de injerto contra hospedero, toxicidad gastrointestinal por fármacos o estados delirantes asociados con la medicación múltiple (no se muestran los datos).

Al realizar el análisis bivariado no se encontró asociación entre la presencia de células endoteliales y la mortalidad, desarrollo de enfermedad de injerto contra hospedero ni enfermedad por CMV. Tampoco se encontró asociación entre los síntomas que presentaban los pacientes y la presencia de células citomegálicas circulantes. Todos los pacientes con antigenemia positiva recibieron terapia antiviral.

\section{Discusión}

El trasplante de órganos sólidos y de médula ósea se ha convertido en tratamiento vital para muchas enfermedades consideradas antes como fatales en nuestro medio. El uso de inmunosupresores es esencial para mantener la función de los trasplantes pero predispone a infecciones por CMV. Por lo tanto, la prevención de la infección por CMV y el manejo oportuno de la enfermedad por CMV se han enfocado en el desarrollo de métodos de diagnóstico, sensibles y específicos, para identificar y tratar oportunamente a los pacientes con la enfermedad establecida o con riesgo de sufrirla.

En un estudio realizado por nuestro grupo, se logró establecer que la antigenemia es una herramienta útil para el seguimiento y el diagnóstico temprano de los pacientes con trasplante de médula ósea. El tratamiento con terapia antiviral preventiva de dichos pacientes con infección activa por CMV, demostrada por 1 célula pp65 positiva, redujo notablemente las complicaciones relacionadas con el CMV (18). Infortunadamente, esta conducta no se puede aplicar por igual a otros grupos de inmunosuprimidos, debido a factores inherentes a cada condición clínica tales como el grado de 
inmunosupresión, el compromiso endotelial variable y la necesidad de terapia antiviral agresiva. Además, como no se ha logrado establecer la gravedad de la infección por medio de la antigenemia, es necesario evaluar otros métodos que permitan precisar la necesidad de tratamiento de acuerdo con la actividad de la infección viral.

La detección de células citomegálicas circulantes ha sido útil para establecer con mayor precisión la relación entre infección por CMV, actividad de la enfermedad y aspectos clínicos asociados. Una vez infectadas, las células endoteliales, blanco de la infección aguda por CMV en pacientes inmunosuprimidos, se desprenden de la membrana basal y entran al torrente sanguíneo, en donde pueden encontrarse durante el pico de antigenemia pp65 (7-9).

Nuestro estudio trató de establecer una posible correlación entre la antigenemia y la detección de células citomegálicas circulantes para diagnosticar actividad de la infección y la enfermedad por CMV. Estudiados 46 pacientes inmunosuprimidos, se encontraron $7(15 \%)$ con antigenemia positiva y 4 ( $9 \%)$ con células citomegálicas circulantes concomitantes. No encontramos asociación estadística entre la presencia de células citomegálicas circulantes y el número de células positivas por antigenemia, la mortalidad, la enfermedad de injerto contra hospedero y la enfermedad por CMV.

Otros autores como Kas Deelen et al. (4) estudiaron la interrelación entre la presencia de células endoteliales citomegálicas y la evolución clínica en 314 muestras de 54 pacientes con trasplante renal. Reportaron la detección de células endoteliales citomegálicas en el $25 \%$ de los pacientes con infección por CMV y una alta correlación entre la presencia de altos recuentos de células endoteliales citomegálicas y el rechazo agudo del órgano trasplantado. De otro lado, Percivalle et al. (12) detectaron altos niveles de células endoteliales citomegálicas en pacientes con trasplante de corazón-pulmón con inmunosupresión farmacológica intensiva, y Slazbeger et al. (13) demostraron bajos niveles de células endoteliales citomegálicas en pacientes con trasplante de médula ósea.
Una de las limitaciones de nuestro estudio fue el número muy bajo de pacientes con transplante renal con antigenemia positiva, apenas 5 de 22; en sólo 3 de ellos (14\%) se detectó la presencia concomitante de células citomegálicas circulantes, por lo cual es difícil correlacionar éstas con antigenemia positiva y evolución clínica de los pacientes. Sin embargo, en nuestros resultados fue obvio que la antigenemia fue mayor en los pacientes con trasplante renal, en los cuales la presencia de células citomegálicas circulantes pareció asociarse, al menos, en 3 de 4 pacientes, con valores altos de antigenemia (cuadro 1).

En este estudio no se pudo demostrar asociación entre la detección de células citomegálicas circulantes, la presencia de antigenemia y la mortalidad. Consideramos que la heterogeneidad de los pacientes incluidos y el bajo tamaño de la muestra son aspectos que influyeron en los resultados encontrados. Además, factores tales como el tipo de trasplante, el grado de inmunosupresión y el tratamiento antiviral pudieron influir no sólo en el curso de la infección citomegálica, sino también en el compromiso endotelial y, por tanto, en el número de células citomegálicas circulantes detectadas.

Aunque existe correlación entre la gravedad de la enfermedad y la carga viral medida por los niveles de antigenemia o ADN viral por PCR, se puede presentar enfermedad con bajos niveles de carga viral, y pueden existir viremias altas en ausencia de enfermedad $(19,20)$. En estos casos, es cuando la detección de células citomegálicas circulantes pudiera tener un impacto significativo en el manejo de los pacientes con trasplante. Los resultados de nuestro trabajo obligan a desarrollar estudios prospectivos con grupos uniformes de pacientes delimitados por su tipo de inmunosupresión, para poder definir en cada grupo los puntos de corte (de células citomegálicas circulantes y antigenemia pp65) relacionados con infección, enfermedad activa y mortalidad por CMV. Ello contribuiría significativamente al mejor cuidado de nuestros pacientes.

\section{Agradecimientos}

Los autores deseamos expresar nuestro agradecimiento a Amado Karduss de la Clínica 
Las Américas por su valiosa colaboración para llevar a cabo este estudio.

\section{Conflicto de intereses}

Los autores manifiestan que no existe ningún conflicto de interés en el desarrollo y publicación de este manuscrito.

\section{Financiación}

Esta investigación fue financiada por el Comité para el Desarrollo de la investigación (CODI) de la Universidad de Antioquia.

\section{Bibliografía}

1. Sia I, Patel R. New strategies for prevention and therapy of cytomegalovirus infection and disease in solid-organ transplant recipients. Clin Microbiol Rev 2000;13:83-121.

2. Crumpacker C. Cytomegalovirus. En: Mandell G, Benett $\mathrm{J}$, Dolin R, editors. Principles and practice of infectious diseases. New York: Churchill, Livingstone; 2000. p.1586-99.

3. Meyers JD, Flournoy N, Thomas ED. Risk factors for cytomegalovirus infection after human marrow transplantation. J Infect Dis 1986;153:478-88.

4. Kas-Deelen AM, de Maar EF, Harmsen M, Driessen C, van Son WJ, The TH. Uninfected and cytomegalic endothelial cells in blood during cytomegalovirus infections: effect of acute rejection. J Infect Dis 2000; 181:721-4.

5. Gerna G, Zavattoni M, Baldanti F, Furione M, Chezzi L, Revello MG et al. Circulating cytomegalic endothelial cells are associated with high human cytomegalovirus (HCMV) load in AIDS patients with late-stage disseminated HCMV disease. J Med Virology 1998;55: 64-74.

6. Gerna G, Percivalle E, Baldanti F, Sarasini A, Zavattoni M, Furione $\mathbf{M}$ et al. Diagnostic significance and clinical impact of quantitative assays for diagnosis of human cytomegalovirus infection/disease in immunocompromised patients. New Microbiol 1998;21: 293-308.

7. Razonable R, Paya CV, Smith TF. Role of the laboratory in diagnosis and management of cytomegalovirus infection in hematopoietic stem cell and solid-organ transplant recipients. J Clin Microbiol 2002;40:746-52.

8. Sinzger C, Grefter R, Plachter B, Gouw A, The TH, Jahn G. Fibroblasts, epithelial cells, endothelial cells and smooth muscle cells are major targets of human cytomegalovirus infection in lung and gastrointestinal tissues. J Gen Virol 1995;76:741-50.
9. Grefte A, Blom N, van der Giessen M, van Son W, The TH. Ultraestructural analysis of circulating cytomegalic cells in patients with active CMV infection. $\mathrm{J}$ Infect Dis 1993;168:1110-8.

10. Fish KN, Soderberg-Naucler C, Mills LK, Stenglein S, Nelson JA. Human cytomegalovirus persistently infects aortic endothelial cells. J Virol 1998;72:5661-8.

11. Boeckh M, Boivin G. Quantitation of cytomegalovirus: methodologic aspects and clinical applications. Clin Microbiol Rev 2000;11:533-54.

12. Percivalle E, Revello L, Vago F, Morini G, Gerna G. Circulating endothelial giant cells permissive for human cytomegalovirus (HCMV) are detected in disseminated HCMV infections with organ involvement. J Clin Inv 1993; 92:663-70.

13. Slazbeger B, Myerzon D, Boeckh M. Circulating CMV infected endothelial cells in marrow transplant patients with CMV disease and CMV infection. J Infect Dis 1997;176:778-81.

14. Bek B, Boeckh M, Lepenies J, Bieniek B, Arasteh $\mathrm{K}$, Heise $\mathbf{W}$ et al. High-level sensitivity of quantitative pp65 cytomegalovirus (CMV) antigenemia assay for diagnosis of CMV disease in AIDS patients and following up. J Clin Microbiol 1996;34:457-9.

15. Grefte A, van der Giessen $\mathbf{M}$, van Son $\mathbf{W}$, The TH. Circulating cytomegalovirus (CMV)-infected endothelial cells in patients with active CMV infection. $\mathrm{J}$ Infect Dis 1993;167:270-7.

16. Bein G, Bitsch A, Hoyer J, Kirchner H. The detection of human cytomegalovirus immediate early antigen in peripheral blood leukocytes. J Immunol Methods 1991;137:175-80.

17. Gerna G, Revello E, Percivalle E, Morini F. Correlation of quantitative human cytomegalovirus pp65, p72 and p150 antigenemia, viremia and circulating endothelial giant cells with clinical symptoms and antiviral treatment in immunocompromised patients. Clin Diagnos Virology 1993;1:47-59.

18. Sarmiento-Maldonado M, Castaño-Orozco M, Bedoya-Escobar VI, Calle-Fernandez S, Velasquez-Lopera M, Gomez-Wolff R et al. Utility of antigenemia test for cytomegalovirus detection in hematopoietic transplant recipients: report of experiences from Medellín, Colombia. Bone Marrow Transplant 2003;32: 119-20.

19. Boeckh M, Bowden RA, Goodarich J, Pettinger M, Meyers JD. Cytomegalovirus antigen detection in peripheral blood leukocytes after allogenic bone marrow transplantation. Blood 1992;80:1358-64.

20. Gerna G, Furione M, Baldanti F, Percivalle E, Comoli P, Locatelli F. Quantitation of human cytomegalovirus DNA in bone marrow transplant recipients. Br J Hematol 1995;91:674-83. 